\title{
Contactless Impedance Cardiography Using Embedded Sensors
}

\author{
E. Pinheiro ${ }^{1}$, O. Postolache ${ }^{2}$, P. Girão ${ }^{3}$ \\ ${ }^{1}$ Instituto de Telecomunicações, Av. Rovisco Pais 1, 10 ${ }^{\text {th }}$ Floor, 1049-001 Lisboa, Portugal, eduardo.pinheiro@lx.it.pt \\ ${ }^{2}$ Instituto de Telecomunicações, ISCTE-IUL, Av ${ }^{\mathrm{a}}$ das Forças Armadas, 1649-026 Lisboa, Portugal, opostolache@1x.it.pt \\ ${ }^{3}$ Instituto de Telecomunicações, Instituto Superior Técnico, Av. Rovisco Pais 1, Lisboa, Portugal, p.girao@1x.it.pt
}

\begin{abstract}
Impedance cardiography is a technique developed with the intent of monitoring cardiac output. By inspecting a few properties of the obtained signal (impedance cardiogram (ICG), the left ventricular ejection time can be derived with certainty, and an estimate of cardiac output is available. This signal is nowadays used in non-invasive monitoring, requiring the placement of electrodes over the subject's skin, either ECG-type or in the form of encircling bands. The work here reported describes the implementation steps and the results obtained when embedding the ICG circuitry in a wheelchair's backrest. The subject is seated normally, is normally dressed, and is completely unaware that monitoring is taking place. That means that the variation of tenths of ohm produced due to the cardiac cycle has to be detected with electrodes having substantial coupling impedance. Contactless ICG with embedded sensors was developed and tested on fourteen healthy subjects. The signal was always acquired, although respiratory activity is also important, constituting a noteworthy innovation in the area.
\end{abstract}

Keywords: Biomedical instrumentation, embedded sensing, impedance measurement, non-invasive monitoring, unobtrusive devices, wheelchair technology

\section{INTRODUCTION}

$\mathrm{T}$ HE IMPEDANCE CARDIOGRAM (ICG) is a representation of the electrical impedance changes in the thorax caused by blood flow. The impedance changes portray a few events of the left ventricle, and strong relations can be established with stroke volume [1].

Knowing the amount of blood ejected by the heart into the aorta is a reliable indicator of disease, and as it is a determinant of blood pressure, it may be an earlier indicator of complications [2].

The electrical impedance measurement technique is the same for practically all implementations, four-terminal impedance measurement [3]-[6]. As a direct current cannot be forced into the body, an alternate current is injected, using frequencies from $20 \mathrm{kHz}$ to $100 \mathrm{kHz}$, because tissues are not excitable in this band. The current amplitude is below $4 \mathrm{~mA}_{\mathrm{RMS}}$ [7]. The electrodes are attached to the chest, and are either bands embracing the subject, or patches as the typical ECG electrodes [2], [8]-[12].

Mostly band-type electrodes are referred to, and from the studies comparing spot and band electrodes [8], [13], it was concluded that although lacking physiological validation of the intended placement positions, spot electrodes provide better sensitivities and lessened artifacts.

In the wheelchair implementation it is not possible to put electrodes over the skin, nor encircling the chest and neck. The spot solution was not studied for placement of the sensing electrodes in the subject's back. Furthermore, embedding spot electrodes makes the position regarding the trunk much more inexact than using an embedded band, while subject posture changes can even miss the electrodes.

Only one approach is known to acquire an impedance signal, in the legs, without electrical contact, by embedding electrodes in a chair seat [6]. Thus contactless and inconspicuous ICG is not a developed approach.

The method projected consisted of placing four parallel metal strips within a cover in the backrest of a wheelchair, and taking the measurements with the subject fully-dressed. That means that the variation of tenths of ohm produced due to the cardiac cycle has to be detected with electrodes having a coupling impedance of hundreds of $\mathrm{k} \Omega$.

As wheelchair users often require extended cardiac accompaniment, this platform was chosen for implementation, but it is implementable in a variety of other elements. The benefits of merely asking the patient to put his back over a covered surface are tremendous both in the time taken in the exam, and in his mental state during the evaluation. Thus an operational circuit is a noteworthy innovation in the area.

The acquisition of the ICG signal by a non-invasive and unobtrusive process has been implemented, and the approaches and limitations of the method are now argued.

\section{ELECTRIC FIELD CREATION}

Unobtrusive measurement of chest impedance changes due to cardiac activity is obtainable in a four-terminal sensing scheme. To acquire the impedance cardiogram a carrier current of tens of $\mathrm{kHz}$ is generated, so that the bioimpedance changes modulate the voltage produced. The approach taken to produce the carrier signal, and demodulate and condition the output of the potential sensed is now reported.

The approach to the measurement is common to all previous implementations [1], [4], [5], [14]. The outer electrodes inject a sinusoidal current, whereas the inner pair senses the voltage produced, which has an amplitude modulation determined by the impedance variations over time. All known implementations of impedance cardiogram, including commercial solutions [11], [15], use direct electric contact.

The elements composing the circuit for creating the electrical field are thus a sinusoidal oscillator and a current source. 


\section{A. Sinusoidal oscillator}

To generate the sinusoidal wave a 3 stage phase shift 63.5 $\mathrm{kHz}$ oscillator was used, since it has been found to have a low-distortion, together with good frequency stability [16]. As current source, the well-known modified Howland circuit was employed [17]. This is a typical circuit to apply in bio-impedance measurements, such as impedance spectroscopy, and multi-frequency electrical impedance tomography [18]. These circuits are displayed in Fig.1., and were implemented with low-noise, low distortion operational amplifiers.

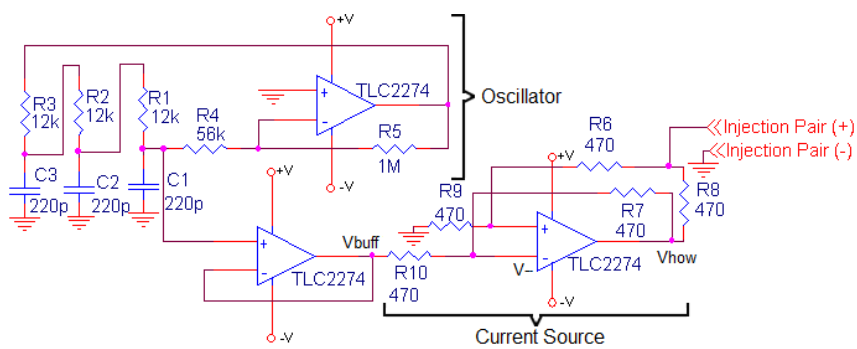

Fig.1. ICG current injection circuitry: sine wave oscillator and current source.
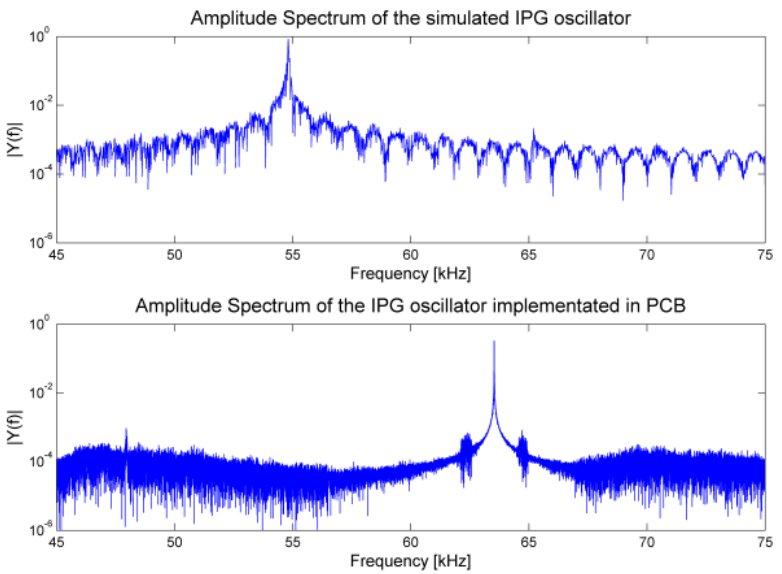

Fig.2. Amplitude spectrum of the ICG sinusoidal oscillator simulation and implementation. Oscillation was projected to occur at $104.4 \mathrm{kHz}$, and Altium Designer simulation provided $54809 \mathrm{~Hz}$ (top), while measurement during one second at $500 \mathrm{kHz}$ from the printed circuit board implementation of the circuit resulted in $63527 \mathrm{~Hz}$ (bottom).

The sinusoidal oscillation is achieved by ensuring the feedback of the amplifier is such that the transfer function is unstable, in other words the Barkhausen criterion [16]. The phase-shift sinusoidal oscillator created is projected to oscillate when each resistor-capacitor (R-C) pair delays the phase $60^{\circ}$, which happens for the conditions of (1). Thus, the expected oscillation frequency for the circuit would be 104.4 $\mathrm{kHz}$.

$$
\tan \left(60^{\circ}\right) / 2 \pi \mathrm{RC} \approx \frac{1.732}{2 \pi 12 \times 10^{3} \times 220 \times 10^{-12}}=104.4 \mathrm{kHz}
$$

Simulation of the circuit provided an oscillation frequency of $54809 \mathrm{~Hz}$. The oscillator implementation on the printed circuit board (PCB) for ICG acquisition was assessed by means of a National Instruments data acquisition board, NI 9201, sampling one second of the signal at $500 \mathrm{kHz}$, and an oscillation frequency of $63527 \mathrm{~Hz}$ was observed. This deviation, although failing by much the projected output frequency, still allowed the circuitry operation, so the sinusoidal oscillator circuit might be revised, but was not. Fig.2. presents the amplitude spectrum, $|Y(f)|$, of the simulated circuit and of its implementation in PCB.

\section{B. Current source}

The modified Howland circuit, by the addition of a fifth resistor (R6 in Error! Reference source not found..), converts the sinusoidal voltage in the output of the operational amplifier buffer, $V_{\text {buff. }}$ The final expression for the current supplied to the load, $I_{L}$, depending also on its voltage, $V_{L}$, is (2)

$I_{L}=\frac{R_{9} R_{7}-R_{10} R_{6}-R_{10} R_{8}}{R_{10} R_{8}\left(R_{9}+R_{6}\right)} V_{L}-\frac{R_{7}}{R_{10} R_{8}} V_{\text {buff }}$

The first term of (2) represents the current drawn that does not flow into the load. Picking the values for the components as $\mathrm{R} 6=\mathrm{R} 7=\mathrm{R} 8=\mathrm{R} 10=\mathrm{R}$, and $\mathrm{R} 9=2 \mathrm{R}$, the cross products nullify the $V_{L}$ term, and the output current will be imposed by the input voltage. The real output impedance of the current source will not be infinite, and will depend on the component values and on the load. As the load will present high capacitive impedance, it is mandatory to simulate the actual circuit behavior.

Simulation proved to be very valuable, and the amplitude curve of the spectral response is presented in Fig.3. The ideal scenario of doubling the $\mathrm{R} 9$ resistor value provided an optimized gain. However, there is a strong decay after 8 $\mathrm{kHz}$. The choice of $\mathrm{R} 9$ equal to every other resistor, symbolized by R9=R, shows a much smaller gain, yet with the benefit of providing a much more stable output, for frequencies up to $1 \mathrm{MHz}$, but most importantly, in the range of interest $50 \mathrm{kHz}$ to $100 \mathrm{kHz}$.
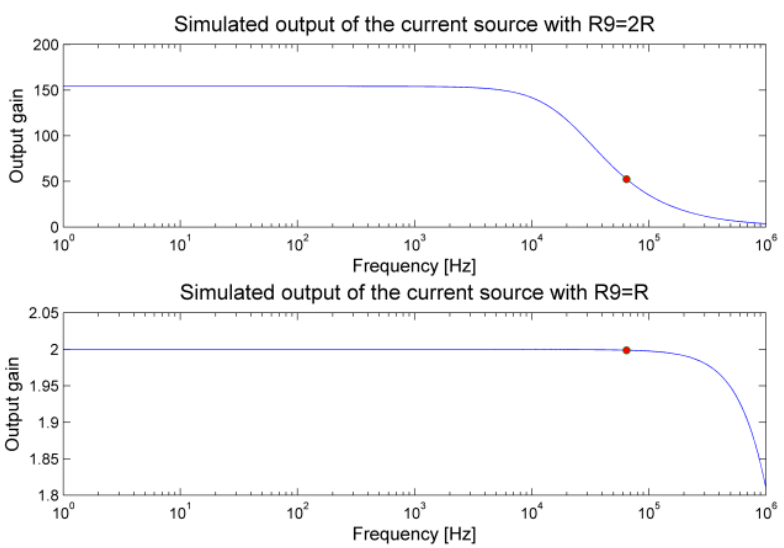

Fig.3. Amplitude spectrum of the ICG current source simulated with two different configurations R9=2R (top) and R9=R (bottom). The frequency of operation is highlighted by a red circle, and it can be seen that it is placed on a steep performance descent of the ideal configuration curve (top), and in a stable performance zone of the R9= R curve (bottom) 
So, the effect of losing the direct relation of $I_{L}$ and $V_{\text {buff }}$ is clearly evident in the gain reduction, but ends up being tolerable as the currents to be drawn are of tens of microamperes, while improving the circuit stability to changes in the frequency regime. Thus, all the resistors will have the same value in the current source. As the sinusoidal generator provides a voltage with $0.71 \mathrm{~V}$ of amplitude, and the current source gain is 2 , the injected current is expected to have $14.2 \mu \mathrm{A}$ of amplitude, for a total injection impedance of $100 \mathrm{k} \Omega$.

\section{ELECTRIC FIELD SENSING}

The abovementioned circuitry creates a $63.5 \mathrm{kHz}$ sinusoidal electric field between the outermost electrodes embedded in the backrest. As a person sits in the wheelchair some current will flow through the skin and a difference of potential can be registered in the inner pair of electrodes.
The subject's clothing and distance to the backrest will be major determinants of the voltage registered, as they define the coupling impedance, and with it the current intensity. The thorax impedance varies, due to blood flow, below $1 \Omega$ with frequency content up to $40 \mathrm{~Hz}$, so there is a demanding voltage demodulation task, requiring a tremendous improvement in signal-to-noise ratio.

The overall circuitry, Fig.4., is composed of a pair of buffers, both followed by a passive first-order $10.6 \mathrm{kHz}$ high-pass filter, feeding the inputs of an instrumentation amplifier with unitary gain. Afterwards, the high-pass filter removes any trace of $50 \mathrm{~Hz}$ and its harmonics interference. After the differential voltage is interfaced, the signal is rectified and filtered, using an op-amp-based precision rectifier with gain 10 , and then passes through a $40 \mathrm{~Hz} 4^{\text {th }}$ order Butterworth low-pass filter, to extract the mean value. Afterwards, it passes five sections implementing first order active band-pass filters to isolate the 0.33 to $21.9 \mathrm{~Hz}$ range, while providing an amplification of $80 \mathrm{~dB}$.

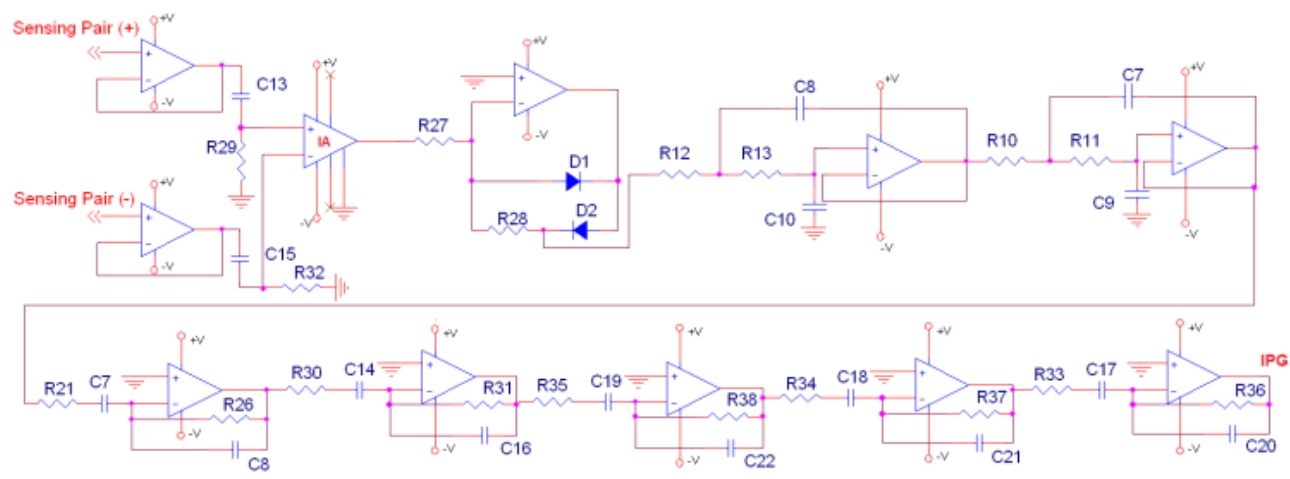

Fig.4. ICG difference of potential sensing circuitry: voltage followers interfacing the electrodes, high-pass filters before the instrumentation amplifier, then a precision rectifier implementing mean value extraction with $4^{\text {th }}$ order $40 \mathrm{~Hz}$ Butterworth low-pass filters, and 0.33 to $21.9 \mathrm{~Hz}$ band-pass active filters with $80 \mathrm{~dB}$ of amplification.

\section{A. Front-end}

The front end of the sensing electrodes is the first problem. It must have very high input impedance, so that the influence of the sensing electrodes coupling impedance, about $92 \mathrm{k} \Omega$ for a separation of $5 \mathrm{~mm}$, using (3), is minimized. Applying a voltage follower in each electrode front end based on TLC2274, allows interfacing the voltage with a greater impedance, and only then computing the difference of potential sensed, with the instrumentation amplifier. As there is interest only in the $63.5 \mathrm{kHz}$ sinusoidal voltage and interferences are always present when sensing low-amplitude potentials (e.g., the power lines), a simple resistor-capacitor $10.6 \mathrm{kHz}$ high-pass filter was added after each buffer. These filters have their cutoff frequency three octaves below the carrier, so they do not disturb the signal, while providing at least $46 \mathrm{~dB}$ of attenuation to the $50 \mathrm{~Hz}$ component. Even if the capacitor tolerances force some mismatch, the separation to the carrier frequency ensures the signal is not affected, only the frequencies of the transition band. After this step, to ensure the voltage arrives at the circuit in proper conditions, the signal can be demodulated.

\section{B. Demodulation}

After the differential voltage appearing at the inner electrodes is interfaced, the signal may be demodulated either by synchronous demodulation, involving a multiplication by the carrier, or by using envelope detectors, involving rectification or filtering operations. The noise limits of affordable multipliers dissuade the usage of carrier multiplication solutions, the second approach. Implementing a mean value extraction scheme with a precision rectifier was preferred.

The voltage to demodulate has a very significant common mode. Since the carrier frequency is $63.5 \mathrm{kHz}$, interference from the other circuits is not the most relevant preoccupation; instead, the signal-to-noise ratio is the problem. Due to the high basal skin impedance, the carrier trace is huge, whereas the modulator, the portion of interest is undersized. The modulation accounts a variation between 0.1 to $0.2 \Omega$ [7], whereas the total skin impedance can be estimated between 125 and $2500 \Omega$ [19].

The stimulus current amplitude is limited by the coupling impedance which can be estimated at $57.6 \mathrm{k} \Omega$, (3), to tens of microamperes. Thus, a difference of potential at most of 
$2500 \times 23.6 \times 10^{-6}=65.75 \mathrm{mV}$ is expected where, in the worst case, $2.36 \mu \mathrm{V}$ are due to the cardiac activity, a signalto-noise ratio of $-88.9 \mathrm{~dB}$, with a signal of a few microvolts of amplitude.

The operational amplifier with R27, R28, D1 and D2 behaves as a half-wave rectifier. When the input voltage is positive the output is the inversion of the voltage amplified by R28/R27, on the contrary, D1 imposes negative feedback to the output, and then the amplifier open loop gain imposes at the output the built-in potential of the diode, $0.7 \mathrm{~V}$, divided by the open loop gain, which is reduced practically to zero. Obtaining an estimate of the modulator is an uncomplicated task, as the ICG signal has a quite limited frequency range, similar to the photoplethysmogram, a $4^{\text {th }}$ order Butterworth low-pass filter of $40 \mathrm{~Hz}$ in Sallen-Key topology was applied.

\section{Last stages}

After demodulation, further amplification and filtering is necessary. Only $20 \mathrm{~dB}$ of gain were given to the acquired voltage up to this stage, and $80 \mathrm{~dB}$ more are applied in 4 sections. As the actual implementation presented a noticeable trace of $50 \mathrm{~Hz}$, the upper limit of the pass band was put at $21.9 \mathrm{~Hz}$, whereas the lower was at $0.33 \mathrm{~Hz}$. A fifth section does not amplify the signal, as it was found that the total of $100 \mathrm{~dB}$ provided was sufficient to create a voltage with reasonable amplitude and able to be acquired without saturating the analog-to-digital converters. Fig.4. shows the sensing circuitry developed for the ICG signal.

\section{EXPERIMENTAL RESULTS AND DISCUSSION}

The embodiment option explored the embedding of four parallel electrodes across the wheelchair backrest beneath the lining, or within a cover, to be perfectly hidden from the user, Fig.5.

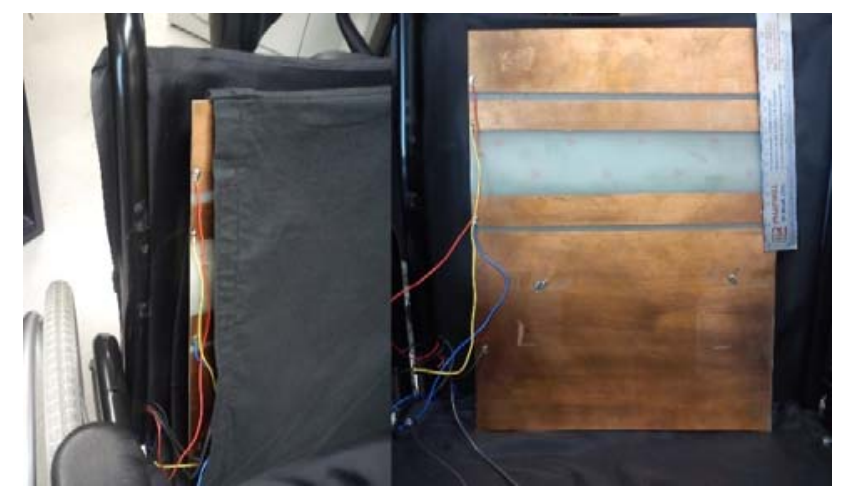

Fig.5. Photographs of the ICG transduction board with a large reference electrode and close sensing electrodes. Built from a single-sided printed circuit board with FR4 substrate (right), and partially embedded in a cover placed over the wheelchair's backrest (left). A ruler with a $20 \mathrm{~cm}$ range is shown for scale.

A simultaneous comparison with a laboratorial impedance meter is not possible. Supplying a second alternate current to the human body, with the same set of electrodes and at the same frequency cannot be achieved. Using a different set of electrodes would impose a disturbance to the developed equipment, whereas the use of a different frequency would change the measurement conditions. Thus, the experimental result section represents a proof of concept of the created instrumentation.

Fourteen subjects, with age between 13 and 56 years, height between 1.50 and $1.88 \mathrm{~m}$, and body mass index between 18.8 and 29.4 tested the implementation.

The recordings were made having in parallel a commercial reference device, Medlab P-OX100. This device provides heart rate and $\mathrm{SpO} 2$ estimates based on PPG from an encapsulating finger grip, and from an ECG from the arms and right leg. This reference equipment does not provide uncertainty evaluation, neither on the waveforms, nor on the estimates it provides. Also, the wheelchair has in its armrests embedded PPG sensors already described [20], to provide a reference plethysmographic waveform.

\section{A. Four thin electrodes}

As the skin to electrode coupling will be without contact, of the capacitive type, the coupling impedance depends on the operation frequency, but will be on the order of tens of $\mathrm{k} \Omega$ for the $20 \mathrm{k}$ to $100 \mathrm{kHz}$. Besides the coupling, the skin impedance alone is about 100 to 1000 times greater than the small pulsate changes to measure [7]. Quantifying, for band electrodes around the ends of the thorax, with direct skin contact, each heartbeat causes an impedance change between 0.1 and $0.2 \Omega$ [7].

Building large injection electrodes is an option towards minimizing the electrode impedance. Several practical implementation issues arise, namely the height at which the electrodes need to be placed to ensure the aorta contribution is captured, and the interest in isolating this contribution to avoid interferences from other physiological processes.

The first development consisted of adding four parallel $\operatorname{sim}(35.6 \mu \mathrm{m})$ and elongated $(300 \mathrm{~mm})$ copper electrodes to the backrest of the wheelchair, in front of an EMFi L-series sensor $(300 \times 290 \mathrm{~mm})$, to simultaneously acquire the ballistocardiogram [21]. The electrodes' height was $50.8 \mathrm{~mm}$ for the injection pair, and $25 \mathrm{~mm}$ for the sensing pair. These electrodes were made of adhesive copper tape, so its placement underneath the wheelchair lining was still imperceptible to the user. Considering clothing with thickness $t$ in millimeters, and relative permittivity 2 [22], the injection total impedance, $Z_{i n i}$, comes from the skin coupling to both electrodes capacitive coupling impedance to the skin. The impedance of each sensing electrode, $Z_{1 s e n}$, comes from the direct application of the capacitor impedance formula. Both $Z_{i n j}$ and $Z_{1 \text { sen }}$ are presented in (3).

$$
\left\{\begin{array}{l}
\left|Z_{\text {in }}\right|=\left(\omega \frac{\varepsilon A_{\text {inj }}}{t}\right)^{-1}+\left(\omega \frac{\varepsilon A_{\text {inj }}}{t}\right)^{-1}+Z_{\text {skin }}=2.5+18.58 t[\mathrm{k} \Omega] \\
\left|Z_{\text {isen }}\right|=\left(\omega \frac{\varepsilon A_{\text {sens }}}{t}\right)^{-1}=18.58 t[\mathrm{k} \Omega]
\end{array}\right\}
$$


The user's freedom in using the wheelchair implies that not even physical contact with the backrest can be insured, and a good improvement over the BCG would be the ICG acquisition even with the user bowing forward. It was seen that the ICG unobtrusively gathered is even more unstable than the ballistic signal. The baseline oscillations are due to subject breathing, and if the subject moves the influence rises.
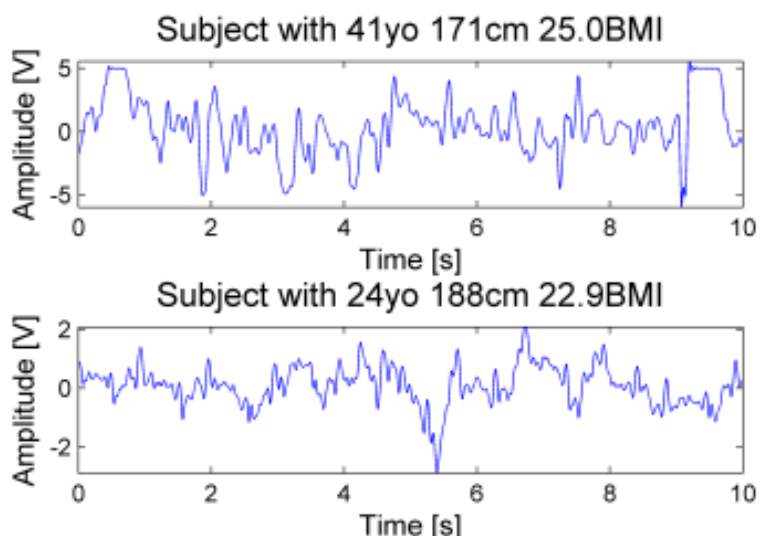

Subject with 41 yo $171 \mathrm{~cm} 29.1 \mathrm{BMI}$

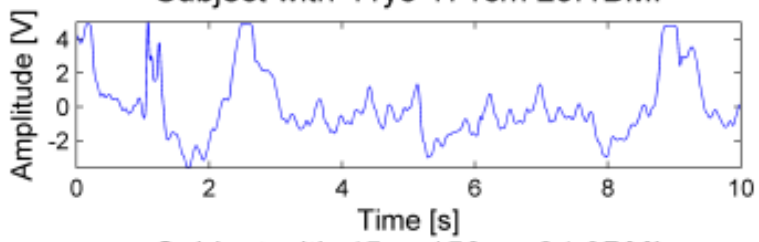

Subject with $45 y$ o $150 \mathrm{~cm} 24.9 \mathrm{BMI}$

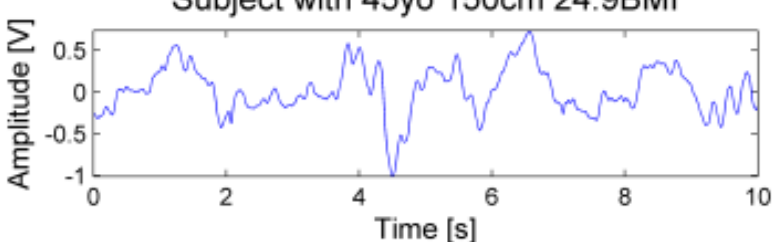

The BCG and ICG signals from the backrest were recorded for eight subjects. A 10 second segment of the recorded ICGs is shown in Fig.6.Error! Reference source not found. In this figure the important influence of breathing is visible, as it defines the baseline in all but one subject. A more detailed view of two of the subjects' ICGs is presented in Fig.7.Error! Reference source not found. These results serve as proof of concept of the feasibility of contactless and embedded ICG acquisition using strip-like electrodes. A new set of electrodes was designed.

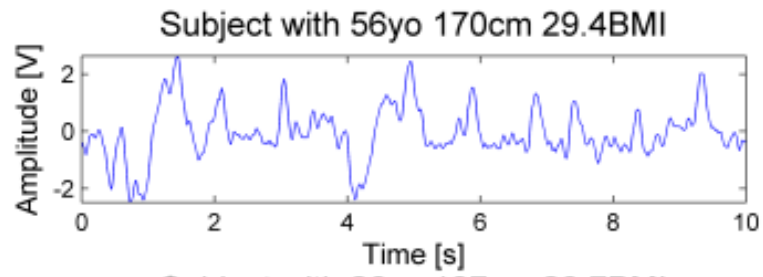

Subject with 26 yo $187 \mathrm{~cm} 23.7 \mathrm{BMI}$

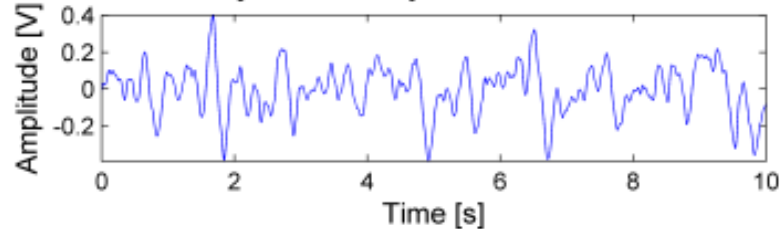

Subject with 13yo $168 \mathrm{~cm} 18.8 \mathrm{BMI}$

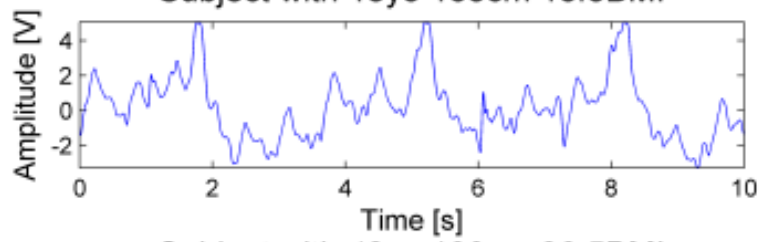

Subject with 42 yo $180 \mathrm{~cm} 26.5 \mathrm{BMI}$

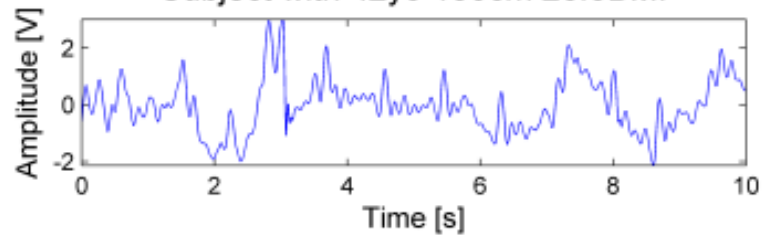

Fig.6. Backrest ICG with copper tape sensors in front of the BCG EMFi sensor within the wheelchair leaning, obtained for eight subjects with the wheelchair stopped. The subjects were dressed in winter clothing. The sensing circuit gain was $127.5 \mathrm{~dB}$, and the electrodes were $20 \mathrm{~cm}$ apart. Baseline oscillations clearly dominate most signals and are related to subject breathing, and breathing-induced motion.
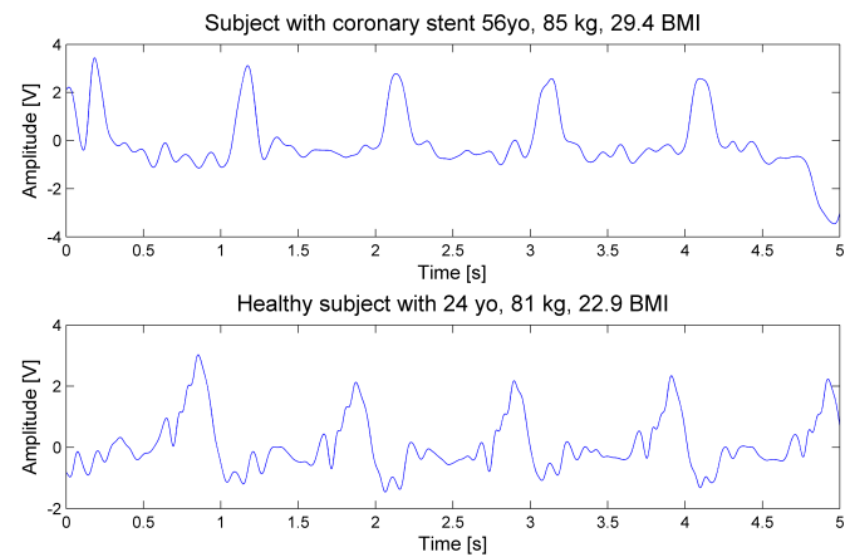

Fig. 7. ICGs gathered from two subjects, a 56 year old subject with a coronary stent (top), and a 24 years old healthy subject (bottom)

\section{B. Final sensing board}

The ICG sensor was then implemented as a stand-alone piece, and it was built of a single-sided printed circuit board with FR4 substrate. This sensing board was shown in Fig.5.

There was no relevance in keeping the maximum exposition area; instead it was preferable to focus on the heart height to diminish the interference caused by blood flow in other tissues and baseline fluctuations due to breathing and other organs. The space freed by the approximation of the inner electrodes was added to the ground electrode of the injection pair to diminish ground impedance, and thus increase the injected current.

ICG signals were obtained for seven more volunteers with the fixed sensing module. From the signals obtained six are exemplified in the signals of Fig.8., and detail of four subjects is given in Fig.9. Clearly, the ICG is obtainable, and it is synchronous with the photoplethysmogram. 


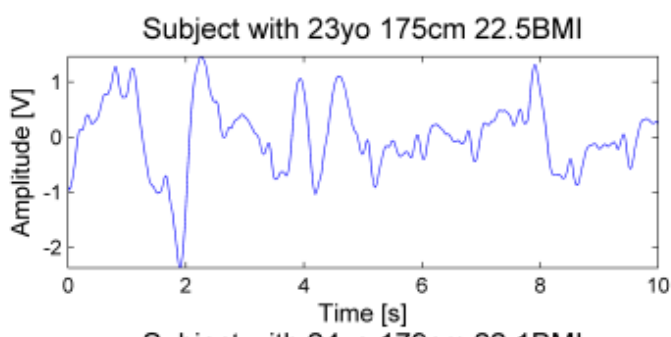

Subject with 24 yo $179 \mathrm{~cm} 22.1 \mathrm{BMI}$

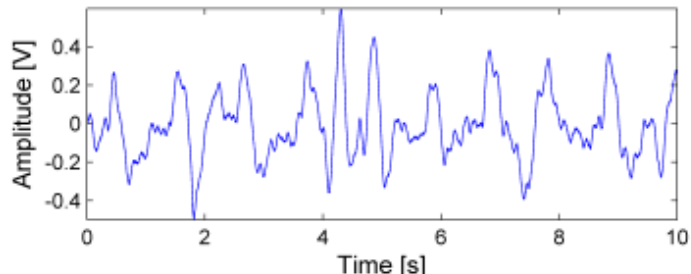

Subject with 25 yo $180 \mathrm{~cm} 19.8 \mathrm{BMI}$

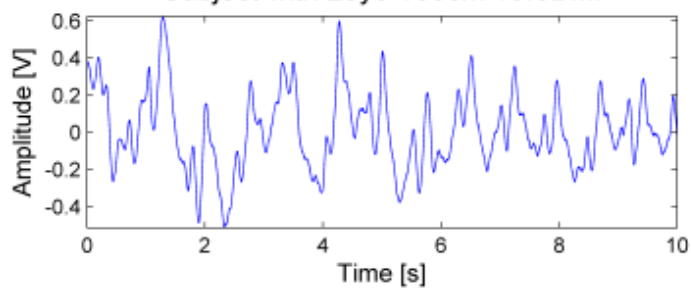

Subject with 44 yo $170 \mathrm{~cm} 27.7 \mathrm{BM}$

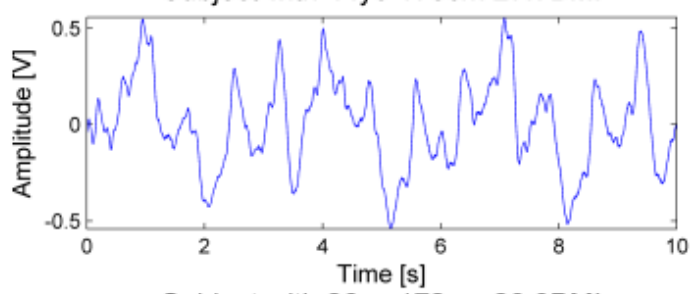

Subject with 26yo $172 \mathrm{~cm} 20.3 \mathrm{BMI}$

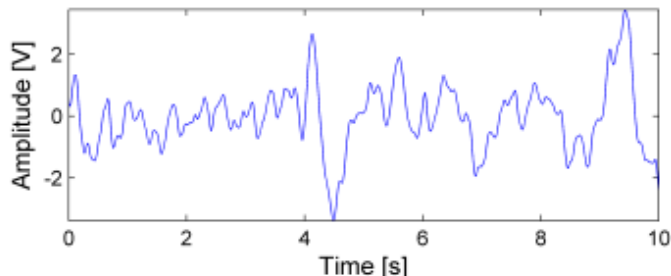

Subject with 26 yo $179 \mathrm{~cm} 24.0 \mathrm{BMI}$

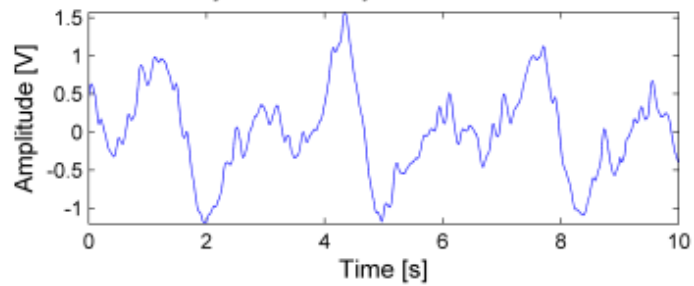

Fig.8. Backrest ICG using the copper board displayed in Fig.5. embedded within the wheelchair cover as sensing module, obtained for six subjects with the wheelchair stopped. The sensing circuit gain was $80 \mathrm{~dB}$. Cardiac component of the signals has quite uniform amplitude.
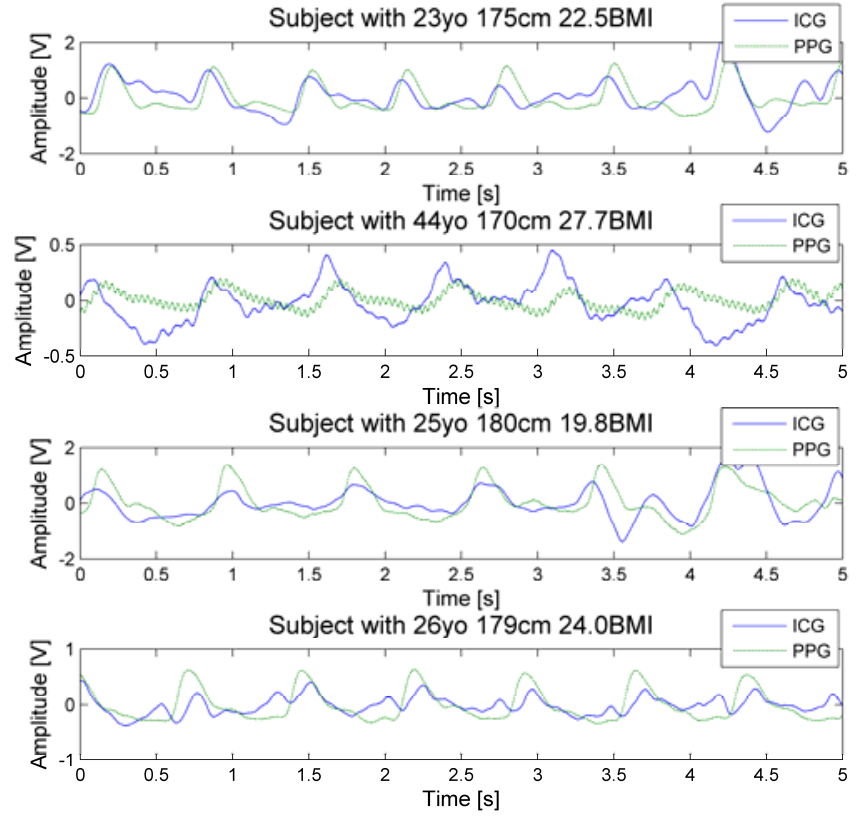

Fig.9. Detail of the backrest ICG together with right hand photoplethysmogram (PPG). The synchronism of the signals is evident, thus stressing the representativeness of the acquired ICG. Results of peak detection present a similar heart rate, especially in the first and second subjects.

The signals' cardiac component has rather constant amplitude with the gain at $80 \mathrm{~dB}$, providing an ICG with amplitude of hundreds of millivolt for all the subjects. Subject breathing provides a few volt of oscillation, without saturating the signal, and less preponderant than in the initial setup. An effective automatic gain control scheme is not easily implementable under these circumstances, as it would act on the respiratory component, since it dominates the ICG signal. It is far preferable to process the signal in order to filter the respiratory component. With such approach the optimal gain value would be around $80 \mathrm{~dB}$ as implemented.

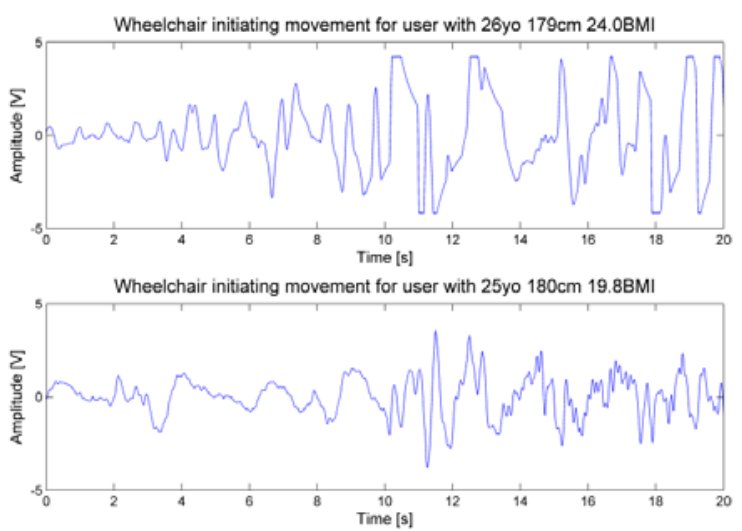

Fig.10. Twenty seconds of backrest ICG using the copper board displayed in Fig.5. embedded within the wheelchair backrest as sensing module, obtained for two subjects when the wheelchair initiates motion. When motion starts, baseline oscillations dominate the ICG. One subject (top) displayed a regular ICG for the first seconds, but it was obliterated during movement and the other (bottom) although avoiding saturation did not produce a visible ICG during motion.

Analyzing movement influence in the signals it can be seen that obtaining a meaningful ICG is extremely difficult. The BCG was found more stable than the ICG, and not useful during motion. The photoplethysmogram is more stable than both, and is almost always able to provide heart 
rate estimates. Being more susceptible than the BCG to subject movement, the ICG vanishes completely during wheelchair propulsion, as the noise created leads to persistent high-amplitude fluctuations of the baseline. An example of this is provided in Fig.10.

\section{Motion influence}

A test session was made for one volunteer on a professional gym treadmill with variable speed and inclination, Pulse Fitness 260FT. Twenty combinations of speed and inclination were experimented with, with the author occupying it, as indicated in Table 1. For each combination, a 5 minute recording was made, and an additional 30 seconds with the wheelchair empty having only the front wheels on the mat.

Table 1. Speeds and inclination at which the wheelchair was recorded.

\begin{tabular}{|l|l|}
\hline Speed $[\mathrm{km} / \mathrm{h}]$ & Inclination $[\%]$ \\
\hline $0.5,1.5,2,2.5,3.5,4.5$ & 0 \\
\hline 1 & $0,1.1,2.2,3.3,4.4$ \\
\hline 3 & $0,1.1,2.2,3.3,4.4$ \\
\hline 5 & $0,4.4$ \\
\hline 7 & $0,4.4$ \\
\hline
\end{tabular}

Six 5-second segments were extracted from each recording, and their RMS value was computed. At speeds below $2 \mathrm{~km} / \mathrm{h}$ the ICGs of the occupied and empty wheelchair RMS values have a correlation of 0.9318 . Fig.11. presents the results obtained.
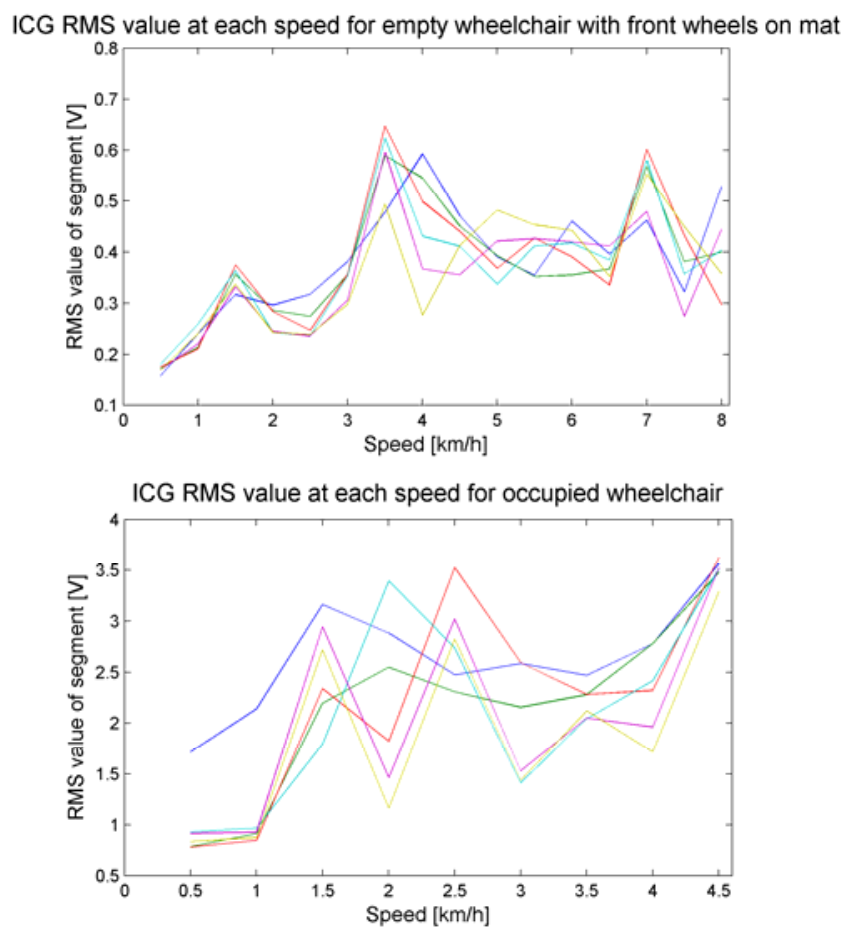

Fig.11. RMS value of all the 5-second segments extracted from the ICG as a function of wheelchair speed. The curves for occupied wheelchair (right), and empty wheelchair with front wheels over the treadmill mat are shown.
This means that, as hinted by the results in Fig.10., the setup created is unable to deal with motion, as the noise generated during movement is the principal explanation of the signal gathered. Faster motion implies greater distortion of the signal, in such a way that there exists a linear relation between speed and RMS value of the signal.

The sensing topology studied and experimentally validated was optimized in terms of spacing, the intended placement regarding the wheelchair user, the minimization of interferences and artifacts, and the maximization of the sensitivity of the circuitry to acquire only cardiac processes. All these elements were taken into account when building the proposed sensing module, and simultaneously while evaluating the circuitry.

\section{CONCLUSIONS}

A valid and completely novel approach to monitor a wheelchair user's impedance cardiogram (ICG), which can be applied to other embedded sensing applications, was described and implemented.

Its behavior is based on the usual ICG measurement scheme of four-terminal sensing. However, the four electrodes were made out of copper strips, and embedded in the wheelchair backrest, thus having to acquire the bioimpedance through the wheelchair lining, and the user's clothes.

The implementation created injects a very weak current of few tens of microamperes. The bio-impedance variation of tenths of ohm modulates the current, and the potential generated in the inner electrodes is demodulated, with the ICG being obtained together with respiratory information.

Comparing with other signals, the ICG acquired by the proposed approach was found less robust to subject noise and respiration than the ballistocardiogram or the photoplethysmogram. Only during very limited motion of the wheelchair, or its passenger, it can be acquired.

Circuitry and the sensing electrodes were thoroughly described, depicted, and the design options were argued. Signals were presented for fourteen subjects with ages between 13 and 56 years, height from 1.50 to $1.88 \mathrm{~m}$, and BMI between 18.8 and 29.4 who tested the acquisition setup, and their signals were presented.

Contactless impedance cardiography using embedded sensors is therefore an achievable goal, and its enabling developments were here reported, and seem promising to the field of unobtrusive cardiac monitoring.

\section{ACKNOWLEDGMENT}

Instituto de Telecomunicações and Fundação para a Ciência e Tecnologia (grant SFRH/BD/46772/2008 and project RIPD/APD/109639/2009) supported the work now reported.

\section{REFERENCES}

[1] Martinsen, O.G., Grimnes, S. (2008). Bioimpedance and Bioelectricity Basics (2nd ed.). Waltham, USA: Academic Press. 
[2] Ventura, H.O., Taler, S.J., Strobeck, J.E. (2005). Hypertension as a hemodynamic disease: The role of impedance cardiography in diagnostic, prognostic, and therapeutic decision making. American Journal of Hypertension, 18 (S2), 26S-43S.

[3] Swanson, D., Webster, J. (1983). Errors in fourelectrode impedance plethysmography. Medical and Biological Engineering and Computing, 21 (6), 674680.

[4] Pallàs-Areny, R., Webster, J.G. (1993). Bioelectric impedance measurements using synchronous sampling. IEEE Transactions on Biomedical Engineering, 40 (8), 824-829.

[5] Patterson, R.P. (1989). Fundamentals of impedance cardiography. IEEE Engineering in Medicine and Biology Magazine, 8 (1), 35-38.

[6] Luna-Lozano, P., Pallàs-Areny, R. (2009). Heart rate detection from impedance plethysmography based on concealed capacitive electrodes. In XIX IMEKO World Congress : Fundamental and Applied Metrology, 6-11 September 2009. IMEKO, 1701-1706.

[7] Patterson, R.P. (2000). Bioelectric impedance measurements. In The Biomedical Engineering Handbook (2nd ed.). Berlin, Germany: Springer.

[8] Qu, M., Zhang, Y., Webster, J.G., Tompkins, W.J. (1986). Motion artifact from spot and band electrodes during impedance cardiography. IEEE Transactions on Biomedical Engineering, 33 (11), 1029-1036.

[9] Van De Water, J.M., Miller, T.W., Vogel, R.L., Mount, B.E., Dalton, M.L. (2003). Impedance cardiography: The next vital sign technology? Chest, 123 (6), 2028-2033.

[10] Muller, M.D., Ryan, E.J., Kim, C.-H., Bellar, D.M., Blankfield, R.P., Glickman, E.L. (2010). Reliability of the measurement of stroke volume using impedance cardiography during acute cold exposure. Aviation, Space, and Environmental Medicine, 81 (2), 120-124.

[11] BIOPAC. Noninvasive cardiac output module NICO100C. http://www.biopac.com/icg-impedancecardiography-noninvasive-cardiac-output-amplifier.

[12] Woltjer, H.H., Bogaard, H.J., de Vries, P.M. (1997). The technique of impedance cardiography. European Heart Journal, 18 (9), 1396-1403.
[13] Kauppinen, P.K., Hyttinen, J.A., Malmivuo, J.A. (1998). Sensitivity distributions of impedance cardiography using band and spot electrodes analyzed by a three-dimensional computer model. Annals of Biomedical Engineering, 26 (4), 694-702.

[14] Valentinuzzi, M.E. (1996). Bioelectrical impedance techniques in medicine. Part I: Bioimpedance measurement. Critical Reviews in Biomedical Engineering, 24 (4), 223-255.

[15] medis. Medizinische Messtechnik GmbH. Niccomo Applications. www.niccomo.com/en/applicat.html

[16] Carter, B., Mancini, R. (2009). Op Amps for Everyone (3rd ed.). Elsevier.

[17] Bertemes-Filho, P., Brown, B.H., Wilson, A.J. (2000). A comparison of modified Howland circuits as current generators with current mirror type circuits. Physiological Measurement, 21 (1), 1-6.

[18] Bertemes-Filho, P. (2002). Tissue characterisation using an impedance spectroscopy probe. Doctoral dissertation, University of Sheffield, Sheffield, UK.

[19] Rosell, J., Colominas, J., Riu, P., Pallàs-Areny, R., Webster, J.G. (1988). Skin impedance from $1 \mathrm{~Hz}$ to 1 MHz. IEEE Transactions on Biomedical Engineering, 35 (8), 649-651.

[20] Pinheiro, E.C., Postolache, O., Girão, P. (2010). Nonintrusive device for real-time circulatory system assessment with advanced signal processing capabilities. Measurement Science Review, 10 (5), 166-175.

[21] Sankaralingam, S., Gupta, B. (2010). Determination of dielectric constant of fabric materials and their use as substrates for design and development of antennas for wearable applications. IEEE Transactions Instrumentation and Measurement, 59 (12), 31223130 .

Received December 17, 2012. Accepted June 10, 2013. 